\title{
Atorvastatin-Induced Hepatotoxicity, Increased by Clopidogrel Stress on CYP450 Enzyme: Understanding the Mechanism through a Case
}

\author{
Sahithi Thotakura ${ }^{1}$, Ajit Singh ${ }^{2}$, Kanav Khera ${ }^{1 *}$, Sheetal Chauhan ${ }^{3}$, Tom Devasia ${ }^{2}$ \\ ${ }^{1}$ Department of Pharmacy Practice, Manipal College of Pharmaceutical Sciences, Manipal Academy of Higher Education, Manipal-576104, India. \\ ${ }^{2}$ Department of Cardiology, Kasturba Medical College and Hospital, Manipal Academy of Higher Education, Manipal-576104, India. \\ ${ }^{3}$ Department of Pharmacology, Melaka Manipal Medical College, Manipal Academy of Higher Education, Manipal-576104, India.
}

\begin{tabular}{|c|c|}
\hline ARTICLE INFO & ABSTRACT \\
\hline $\begin{array}{l}\text { Article history: } \\
\text { Received on: } 12 / 01 / 2018 \\
\text { Accepted on: 20/02/2018 } \\
\text { Available online: } 29 / 04 / 2018\end{array}$ & $\begin{array}{l}\text { Statins are widely used drugs for both primary and secondary prevention of various cardiovascular disorders. Cases } \\
\text { have been reported on statin-induced hepatitis where the elevation of liver enzymes leads to hepatotoxicity. Here we } \\
\text { present a patient with atorvastatin-induced hepatotoxicity and the mechanism accelerated by clopidogrel use. The } \\
\text { drugs which are metabolized by CYP } 450 \text { can increase the risk of hepatitis. CYP4503A4 enzyme metabolizes both } \\
\text { atorvastatin and clopidogrel. In this case, clopidogrel has shown the synergistic effect which is responsible for the }\end{array}$ \\
\hline $\begin{array}{l}\text { Key words: } \\
\text { Hepatotoxicity, Coronary } \\
\text { artery disease, Alanine } \\
\text { aminotransferase, } \\
\text { Aspartate aminotransferase, } \\
\text { Medication non-compliance, } \\
\text { RUCAM Score. }\end{array}$ & \\
\hline
\end{tabular}

\section{INTRODUCTION}

Statins, the hydroxymethyl glutaryl CoA (HMG-CoA) inhibitors are widely used drugs for both primary and secondary prevention of various cardiovascular disorders (Otto, 2016). Statins are usually safe and can cause an elevation in transaminases in around $3 \%$ of the users. Cases have been reported on statininduced hepatitis where the elevation of liver enzymes lead to liver dysfunction ranging from reversible to irreversible liver injury, autoimmune hepatitis, hepatocellular injury and even death in a few cases (Clarke and Mills, 2006; Vishwakarma et al., 2014). High dose of statin and old age may increase patient susceptibility to statin-induced liver injury. Among the statin-induced hepatitis cases, atorvastatin and simvastatin showed more incidences of

*Corresponding Author

Kanav Khera, Department of Pharmacy Practice, Manipal College

of Pharmaceutical Sciences, Manipal Academy of Higher Education,

Manipal-576104, India.

E-mail:kanavworld@gmail.com hepatic damage (Björnsson, 2017).

Idiosyncratic liver injury with statins is rare and can be severe after recovery on re-exposure. Here, we present a case of statin-induced reversible liver injury and its clinical summary, progress, and management.

\section{CASE PRESENTATION}

A 61-year-old male presented to the medicine department of a tertiary care center with chief complaints of fever with moderate chills for 2 weeks, two episodes of vomiting, nausea, upper segment abdomen pain and severe fatigability for 1 week. He had a history of type 2 diabetes mellitus (T2DM) for 2 years and continuing the oral hypoglycaemic agent, glimepiride.

He had ischemic heart disease (IHD)/anterior wall myocardial infarction (AWMI) six months back. Coronary angiogram (CAG) showed 100\% thrombotic occlusion of proximal left anterior descending (LAD) artery, total occlusion of mid left circumflex (LCx) artery. Percutaneous coronary angioplasty (PTCA) was done, and drug-eluting stent was placed 
to the proximal LAD. He also developed heart failure with reduced ejection fraction; echocardiography (ECHO) showed mild to moderate left ventricular dysfunction with ejection fraction, $43 \%$. For coronary artery disease (CAD) the patient was started on antiplatelet drugs (aspirin $150 \mathrm{mg}$ daily and clopidogrel 75 $\mathrm{mg}$ daily) along with atorvastatin (40 $\mathrm{mg}$ daily) and discharged on the same dose of antiplatelet medications with a low dose of atorvastatin $(10 \mathrm{mg})$. Furosemide $40 \mathrm{mg}$ and spironolactone 25 $\mathrm{mg}$ daily along with digoxin $0.125 \mathrm{mg}$ daily was continued for heart failure treatment. Telmisartan $40 \mathrm{mg}$ daily was started for newly detected hypertension and glimepiride for type 2 diabetes mellitus (T2DM). Aspirin reduced to $75 \mathrm{mg}$ per day along with previous dose of clopidogrel and atorvastatin on 1 month follow up after angioplasty.

He is a reformed alcoholic and non-smoker. He does not have any history of jaundice or any other hepatic illness.

At present admission, presence of tenderness in right upper quadrant supported jaundice. His vitals were normal, and further biochemical investigations were done in view of suspected liver injury. Serum electrolytes, creatinine, urea, RBS, HbAlc were normal. His liver enzymes were deranged at admission as aspartate aminotransferase (AST), alanine transaminase (ALT), alkaline phosphatase (ALP) and direct bilirubin were $315 \mathrm{IU} / \mathrm{L}$, $452 \mathrm{IU} / \mathrm{L}, 109 \mathrm{U} / \mathrm{L}$ and $1.3 \mathrm{mg} / \mathrm{dL}$ respectively. The patient was screened for acute viral hepatitis. Antibodies for hepatitis A and $\mathrm{C}$ virus, antibodies for hepatitis $\mathrm{B}$ surface and core antigen were non-reactive. USG abdomen and pelvis showed borderline hepatomegaly with grade 1 fatty infiltration, no obvious focal lesions, no intrahepatic duct dilation but bilateral renal calculi. The patient was suspected to have statins induced hepatotoxicity so atorvastatin was stopped and liver enzymes were monitored. After 5 days the liver enzymes came back to normal and were noted normal at 60 days follow up after statin withdrawal (Table 1). Statins were not re-initiated till recent follow up.

Table 1: The table shows the variation of liver enzyme levels before initiation and after the withdrawal of the statin.

\begin{tabular}{llll}
\hline & AST & ALT & ALP \\
\hline $\begin{array}{l}\text { Prior to statin initiation (during hospitalization } \\
\text { for IHD) }\end{array}$ & 25 & 23 & 96 \\
After 6 months of statin use (at recent admission) & 315 & 452 & 109 \\
2 days after stopping statins & 99 & 282 & 99 \\
5 days after stopping statins & 40 & 130 & 92 \\
60 days after stopping statins & 21 & 22 & 99 \\
\hline
\end{tabular}

He is not adhering to medications and left heart failure medicines for few weeks. He also developed dyspnoea on exertion (NYHA grade III) during present hospitalization. Signs of acute pulmonary oedema and paroxysmal nocturnal dyspnoea suggested the acute worsening of heart failure symptoms. Bilateral basal crepts were heard, and bilateral pedal oedema was seen on physical examination. Blood pressure and heart rate were noted normal. Troponin T was normal $(<0.01 \mathrm{ng} / \mathrm{mL})$, but NT-proBNP was elevated $(4398 \mathrm{pg} / \mathrm{mL})$ which confirmed the acute worsening of heart failure. ECHO showed severe left ventricular dysfunction with ejection fraction $27 \%$, moderate mitral regurgitation and moderate to severe tricuspid valve regurgitation, moderate pulmonary arterial hypertension accompanied by moderate right ventricular dysfunction. An apical clot of $2.4 \times 1.6 \mathrm{~cm}$ was also seen in the echocardiogram. During hospitalization, the patient was managed with intravenous diuretics (furosemide $40 \mathrm{mg} \mathrm{Q8H}$ for 3 days), positive inotropes (dobutamine $5 \mathrm{mcg} / \mathrm{kg} / \mathrm{min}$ for 48 hours). Pulmonary oedema was subsided with diuretics and patient improved. At 2 months follow up patient showed improvement in heart failure symptoms.

\section{DISCUSSION}

The patient was suspected of drug-induced liver injury (DILI) as other causes were ruled out. The assessment of drug-induced liver injury is done by calculating the Roussel Uclaf Causality Assessment Method (RUCAM) for adverse drug reactions (ADR). In the existing therapy clopidogrel and atorvastatin were known to cause hepatitis. The RUCAM score was done for atorvastatin and found to be probable (Score 4) ADR. As there are few cases present in the literature, supporting statin-induced hepatitis. Initially, the statin was stopped, and the liver enzymes turned to normal levels.

Atorvastatin-induced acute liver injury was reported in a patient with stroke and hyperlipidemia who was continuing $20 \mathrm{mg}$ of the drug (Vishwakarma et al., 2014). Two cases of atorvastatin associated acute elevation in liver enzymes were reported from China (Liu et al., 2010). The U.S. Drug-Induced Liver Injury Network has done a registry on 1188 cases of drug-induced liver injury (DILI) between 2004 and 2012, where they found only $1.8 \%$ (22) patients attributed to statins. Only 3 cases showed atorvastatin-induced hepatotoxicity in above registry (Russo et al., 2014).

The process/mechanism takes place by blocking the conversion of HMG-CoA to a precursor of cholesterol (mevalonate) by competitively inhibiting HMG-CoA reductase enzyme; a step in biosynthesis of cholesterol (Vishwakarma et al., 2014).

Risk factors for atorvastatin-induced liver injury are old age, female gender, genotypes of drug metabolizing enzymes and co-medications (e.g., ketoconazole, erythromycin etc.). The suggested mechanisms of statin-induced liver injury were functional CYP3A polymorphisms, inducers/inhibitors of CYP4503A enzyme and autoimmune phenotype (Chen et al., 2015).

Drug-drug interactions are one of the possible risk factors for statin-induced hepatitis (Jacobson, 2004). The drugs which are metabolized by CYP450 can increase the risk of hepatitis. CYP4503A4 enzyme metabolizes both atorvastatin and clopidogrel. CYP4503A activates clopidogrel into its active metabolite whereas this enzyme primarily metabolizes atorvastatin and this increases the stress on the enzyme which may cause an increase in the liver enzymes levels leading to druginduced hepatitis (Monteiro et al., 2011). In this case, clopidogrel has shown the synergistic effect which is responsible for the significant liver injury.

Penetration of the drug into organs depends upon the lipophilicity of the drug. Stains are metabolized in different ways. Atorvastatin, Simvastatin, lovastatin are lipophilic and extensively undergo hepatic metabolism. These increase the smooth muscle proliferation and have a high incidence of myalgia, so drug interactions are more. Rosuvastatin and pravastatin are 
hydrophilic and do not undergo hepatic metabolism. Pravastatin is converted into metabolites via chemical degradation and doesn't involve hepatic activation. It is eliminated via renal and nonrenal routes (Hatanaka, 2000). Rosuvastatin is not extensively metabolized by the liver and doesn't show interactions with CYP4503A4 inhibitors and shows little interaction with CYP2C9 inhibitors. Rosuvastatin has less affinity towards CYP4503A4 enzyme (Martin et al., 2003).

Cases reported with pravastatin and rosuvastatin induced hepatitis is less when to other statin and fatal liver injury has not found in literature. Though trails on safety and efficacy of statins included a large group of people in the study, they couldn't discover the rare side effects of statins such as hepatic injury (Björnsson, 2017).

The patient also had a mild to moderate LV dysfunction at first admission but worsened to severe LV dysfunction with NYHA class III symptoms. It is because of medication nonadherence. Medications were re-introduced at present admission, and heart failure symptoms came down at follow up.

\section{CONCLUSION}

Statin-induced hepatitis is rare but can be severe. There are cases reported with a reversible liver injury to fatal hepatotoxicity. FDA label changes of statin suggest measuring liver enzymes before initiating statin therapy that can prevent severe hepatotoxicity. But in the presence of drugs that increase the risk of periodic hepatitis, monitoring of liver enzymes can prevent the further injury.

\section{INFORMED CONSENT}

Informed consent was taken from the patient before examination and publication.

\section{REFERENCES}

Björnsson ES. Hepatotoxicity of statins and other lipid-lowering agents. Liver International. 2017; 37(2):173-8.

Chen M, Suzuki A, Borlak J, Andrade RJ, Lucena MI. Druginduced liver injury: Interactions between drug properties and host factors. Journal of hepatology. 2015; 63(2):503-14.

Clarke AT, Mills PR. Atorvastatin associated liver disease. Digestive and liver disease. 2006; 38(10):772-7.

Hatanaka T. Clinical pharmacokinetics of pravastatin. Clinical pharmacokinetics. 2000; 39(6):397-412.

Jacobson TA. Comparative pharmacokinetic interaction profiles of pravastatin, simvastatin, and atorvastatin when coadministered with cytochrome P450 inhibitors. American Journal of Cardiology. 2004; 94(9):1140-6

Liu Y, Cheng Z, Ding L, Fang F, Cheng KA, Fang Q, Shi GP. Atorvastatin-induced acute elevation of hepatic enzymes and the absence of cross-toxicity of pravastatin. International journal of clinical pharmacology and therapeutics. 2010; 48(12):798.

Martin PD, Warwick MJ, Dane AL, Hill SJ, Giles PB, Phillips PJ, Lenz E. Metabolism, excretion, and pharmacokinetics of rosuvastatin in healthy adult male volunteers. Clinical Therapeutics. 2003; 25(11):2822-35.

Monteiro PH, Pinheiro LD, Alvoeiro L, Lucas M, Victorino RM. Clopidogrel-induced liver failure. JRSM short reports. 2011; 2(5):1-3.

Otto C. Statins for primary prevention of cardiovascular disease. BMJ. 2016; i6334.

Russo MW, Hoofnagle JH, Gu J, Fontana RJ, Barnhart H, Kleiner DE, Chalasani N, Bonkovsky HL. Spectrum of statin hepatotoxicity: Experience of the drug-induced liver injury network. Hepatology. 2014; 60(2):679-86.

Vishwakarma P, Nehra R, Kumar A. Acute hepatic injury with atorvastatin: An unusual occurrence. Indian journal of pharmacology. 2014; 46(3):343.

How to cite this article:

Thotakura S, Singh A, Khera K, Chauhan S, Devasia T. Atorvastatin-Induced Hepatotoxicity, Increased by Clopidogrel Stress on CYP450 Enzyme: Understanding the Mechanism through a Case. J App Pharm Sci, 2018; 8(04): 168-170. 